symmetrical case with $n=6$. The second was taken from the paper by Brock and Murray, with $n=3$ and the $\alpha_{i}$ consisting of two pairs of complex conjugates. Using the full 10-figure capacity of a desk machine, cancellation reduced the accuracy of the results obtained to some four significant figures in the first example and six in the second. The procedure given here should, however, be readily adaptable to electronic digital computers, and the increased capacity of those machines should enable the coefficients $B(k, m, n ; h, r)$ to be calculated to any accuracy likely to be required in practice.

Sevenoaks

Kent, England 352-359.

1. W. G. Bickley, "Formulae for numerical integration," Math. Gaz., v. 23, 1939, p.

2. R. E. GREENWOOD, "Numerical integration for linear sums of exponential functions," Ann. Math. Statist. v. 20,1949, p. 608-611.

3. P. Brock \& F. J. MURRAY, "The use of exponential sums in step by step integration," $M T A C$, v. 6,1952, p. $63-78$ and $138-150$.

\title{
New Factors of Mersenne Numbers
}

\section{By Edgar Karst}

I have tested for prime factors all the Mersenne numbers $2^{p}-1$ corresponding to prime exponents $p$ in the interval $3000<p<3500$. The limit of the search for factors was $9 p^{2}$ when no factor was previously known; otherwise the limit was $3 p^{2}$.

The nineteen new prime factors of Mersenne numbers found by this search are displayed in the following table. Factors corresponding to smaller values of $p$ have been listed in a paper by Brillhart and Johnson [1].

\begin{tabular}{|c|c|}
\hline$p$ & New factors of $2^{p}-1$ \\
\hline 3037 & 145777 \\
\hline 3041 & 5565031 \\
\hline 3067 & 22063999 \\
\hline 3083 & 15914447 \\
\hline 3119 & $230807 \cdot 14222641$ \\
\hline 3121 & 31509617 \\
\hline 3167 & 12237289 \\
\hline 3181 & 127241 \\
\hline 3191 & $4089585 \pi$ \\
\hline 3253 & $464528+1$ \\
\hline 3257 & $\begin{array}{lll}4032 & 167\end{array}$ \\
\hline 3299 & 19873177 \\
\hline 3329 & 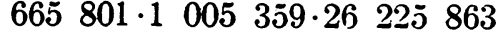 \\
\hline 3391 & $\begin{array}{r}1519169\end{array}$ \\
\hline 3433 & $\begin{array}{lllll}5 & 952 & 823 \cdot 12 & 688 & 369\end{array}$ \\
\hline
\end{tabular}

An extensive table by Riesel [2] includes smaller prime factors of Mersenne numbers corresponding to $p=3037,3041,3119,3121,3181,3257,3299,3329$, 3391 , and 3433 in the preceding table.

\section{Brigham Young University}

Provo, Utah

1. John Brillhart \& G. D. Johnson, "On the factors of certain Mersenne numbers," Math. Comp., v. 14, 1960, p. 365-369.

2. H. RIESEL, "Mersenne numbers," MTAC, v. 12, 1958, p. 207-213.

Received September 14, 1959; in revised form July 26, 1960. 